\title{
DECLINE AND APPROACHES TO REVITALIZATION IN SMALL CITIES: THE CASE OF SAGA, JAPAN
}

\author{
Munetoshi Yamashita \\ Faculty of Culture \& Education, Saga University, Japan \\ Saga 840-8502, Japan \\ e-mail:yama@cc.saga-u.ac.jp
}

\begin{abstract}
The study of urban geography has primarily focused on large cities, but geographic trends affecting smaller urban areas also merit the attention of researchers. The local economies of smaller municipalities in Japan, for example, have been more seriously damaged by the recession of the last decade than the economies of larger cities. Typically, store vacancies have increased in small cities as a result of the recession. Moreover, the population of small cities is aging rapidly, obscuring their prospects for the future.

This paper examines the outcome of changes in the case of the small city of Saga, Japan, with particular attention to the decline of its urban center and its unsuccessful efforts at downtown revitalization. As the center of Saga Prefecture in southwestern Japan, the city of Saga performs communication, education, administrative, and cultural functions that are significant in the local and regional economy. As in other small cities in Japan, the downtown of Saga has declined with the regional economy in recent years. The outlook for Saga's urban environment is serious, with increasing urban problems anticipated. In response, downtown revitalization and redevelopment efforts have been undertaken, but these have been unsuccessful. This study concludes that a lack of both public participation and clear policy purpose were contributing factors in the failure of Saga's revitalization efforts.
\end{abstract}

Key words: decline, revitalization, small city, Saga, Town Management Organization

\section{INTRODUCTION}

While the study of urban geography has focused to a remarkable degree on large cities, with major metropolises such as Chicago, Los Angeles, London, Paris, and Tokyo providing the cases for theories of urban geography, geographic trends involving smaller cities have been of little concern to researchers. Yet small- and medium-sized cities are home to the great majority of municipalities in Japan, for example, whereas cities with populations 
exceeding one million number only twelve ${ }^{1}$. Furthermore, small cities in particular face issues of progress that in many respects are more serious than those confronting large cities. The state of urban centers in small cities should thus be paid greater attention. With this in mind, this paper examines the case of a small city in Japan, the city of Saga, focusing on Saga's decline during the current economic recession as well as its unsuccessful efforts to revitalize its downtown.

As an initial step in this case study, let us briefly consider the context of Saga's urban decline, beginning with the recent economic recession as it affects the city. The inflation of the economic bubble in the 1980s led to rapid increases in real estate prices; as is well known, the collapse of the bubble around 1990 resulted in a tremendous burden of bad debt for banking institutions. This burden along with mismanagement of the bad debt was a major cause of the recent economic recession, which has been characterized by commercial decline in city centers as well as more general urban decline. The bubble economy, therefore, has been particularly deleterious for some downtown land uses, such as small extensive land use, which are common in downtown Tokyo (Yamashita, 2002). The post-bubble recession, moreover, has also augmented population drains from city centers, another cause of urban decline.

In response to the economic stagnation that has followed the collapse of the bubble economy, the central government of Japan has vigorously pushed forward urban redevelopment projects in largest cities to stimulate the economy. In the metropolitan core of Tokyo, numerous high-rise buildings have been constructed; one of the latest such large-scale redevelopment projects, for example, is Roppongi Hills ${ }^{2}$, a complex encompassing approximately 760 thousand square meters of floor space for both commercial and residential. A number of large-scale redevelopment projects like Roppongi Hills have recently been completed one after another.

In contrast to the metropolitan core of Tokyo, smaller cities have received little attention from the central government; with the exception of Tokyo, in other words, Japan's cities are struggling for urban revitalization against mountains of urban problems.

Taking up the case of Saga, Japan, Section 2 of this paper presents the characteristics of the study area. In Section 3, efforts that were undertaken to revitalize Saga's downtown are investigated. In Section 4, a comparative discussion is offered of management practices and the public input process in particular revitalization approaches: specifically, those of Town Centre Management (TCM) in the United Kingdom, of the Business Improvement District (BID) in the USA, and of the Town Management Organization (TMO) in Japan.

\footnotetext{
${ }^{1}$ Number of cities with over one million populations amounts to twelve in Japan. They cover $0.32 \%$ only of all municipalities which include Shi, Machi, and Mura. Tokyo 23Wards: 8,135 thousand, Yokohama: 3,427 thousand, Osaka: 2,599 thousand, Nagoya: 2,172 thousand, Sapporo: 1,822 thousand, Kobe: 1,493 thousand, Kyoto: 1,468 thousand, Fukuoka: 1,341 thousand, Kawasaki: 1,250 thousand, Hiroshima: 1,126 thousand, Kita-Kyushu: 1,011 thousand, Sendai: 1,008 thousand. Source: Population Census 2000

${ }^{2}$ Roppongi Hills is called a multi-purpose city within a city. Besides office space, the newly completed complex includes a tower with an art gallery, a library, and an observation deck; a nine-screen cinema complex with seating for 2,100 people; a hotel complex containing the Grand Hyatt Tokyo and numerous first-class restaurants; an event arena and garden complete with a circular stage; four residential apartment buildings (total 800 units) that boast a highly sought after city-center location; and the headquarters of a major TV station.
} 
In conclusion, the results of the study are summarized and their policy implications are discussed.

\section{THE CITY OF SAGA}

This section provides an overview of the urban geography of Saga, capital of Saga Prefecture, Japan. In the first subsection, a description of Saga in terms of the historical urban structure of the city, its administrative and commercial functions, and other geographic phenomena in progress is offered. In the second subsection, the decline of Saga's downtown area is characterized. In the third subsection, factors contributing to this decline are identified.

\section{Saga: An Introduction}

As the prefectural capital of Saga Prefecture, the city of Saga has the highest level of urban function. According to the 2000 census, the population of the entire municipality includes 63,637 households, of which about one fifth, comprising a population of 167,955 , live in the city of Saga. Densely Inhabited Districts (DIDs) ${ }^{3}$ in Saga cover 23.81 square kilometer, with a total population of 127,010 people; the population density of DIDs is thus $5,334.3$ people per square kilometer. As a result of outward expansion through development on the outskirts of the city, this DIDs population density has decreased in recent years. An ongoing economic recession has also accentuated the decline of the downtown population of Saga. The city's rank of 136 on a rank-size graph of cities with the thousand highest DID populations in Japan shows that Saga is nothing but a typical local city (Figure 1).

As shown in Figure 2, a land use map created using Geographic Information Systems (GIS), most of the built-up areas of Saga are located on lowlands which are less than five meters above sea level, and are surrounded by paddy fields. The Central Business District (CBD) of Saga, comprising such typical features as tall buildings, crowds of pedestrian, well-established department stores, shops, banks, theaters, and other commercial land uses, may be observed to the north of the Saga castle. The CBD in Saga is not so larger than those of other prefectural capitals in Japan.

The urban centrality of Saga is not so high, however, Saga has developed as the center. There are some reasons rooted in the long history of the city, which was originally an old castle town in the Edo Period. In that era, the old Nagasaki Road connecting the port of Nagasaki and the city of Kokura in Fukuoka Prefecture passed through Saga's downtown;

\footnotetext{
${ }^{3}$ Densely Inhabited Districts (DIDs) were established for the first time in the 1960 Population Census in Japan, taking into consideration the large-scale merger of shi, machi, and mura in the preceding years which had made the urban-rural classification obscure. A DID is defined as an area, (1) using census -enumeration districts as the basic unit of area, (2) composed of a group of contiguous census enumeration districts with high population density (in principle, 4,000 inhabitants or more per square kilometer) within the boundary of a shi (city), ku (ward), machi (town) or mura (village), (3) constituting an agglomeration of 5,000 inhabitants or more as of the date of the census.
} 
this road served as a conduit through which foreign culture and technology entered Japan, and Japanese culture departed for foreign shores. The old road has been running in a narrow zigzag around the Saga castle even now, and the city's downtown has developed along its path. This downtown has subsequently flourished as the main regional center of the Saga area.

Figure 1: Rank-size graph for DIDs in Japan (2000)

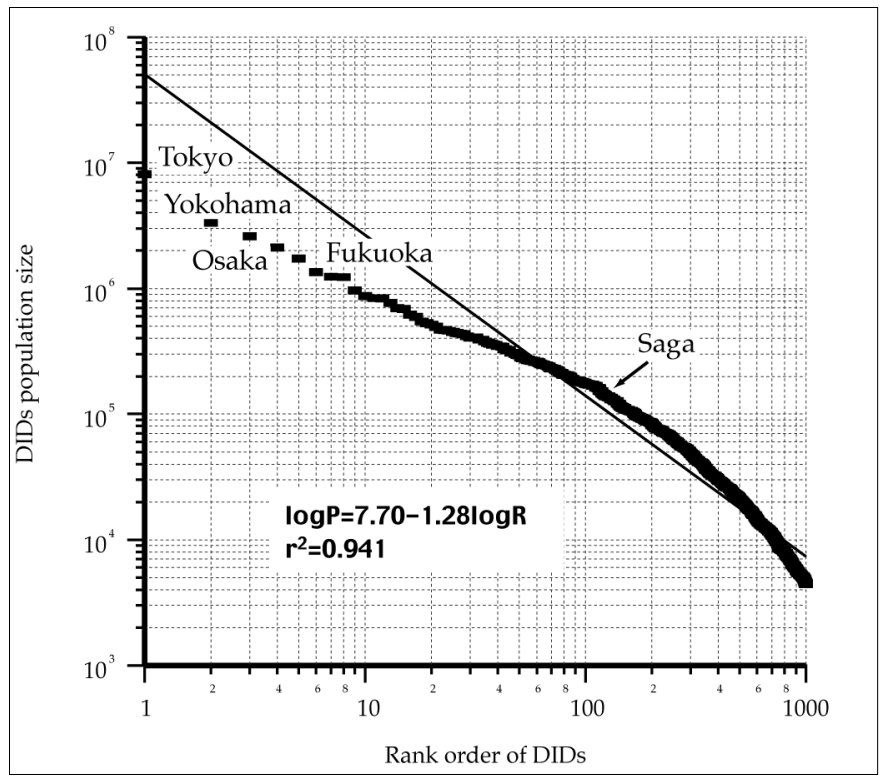

Source: Population census of Japan

\section{The decline of downtown Saga}

Since the early 1990s, however, Saga's downtown has undergone a decline in a number of respects. First, the downtown has experienced a population decline that has left the streets deserted and has led to an elevated proportion of the elderly among the remaining inhabitants. The 25,431 people aged 65 or over who reside in the city of Saga according to the 2000 census constitute $14.9 \%$ of the city's overall population, but a much higher percentage of the population of downtown districts.

In Figure 3, the percentage of the population aged 65 or over in 2000 is shown by district $^{4}$; the spatial distribution of areas with higher or lower proportions of the elderly in three concentric zones reveals the geographical phenomenon of suburban sprawl and aging. First, the core of downtown Saga, which covers about three square kilometers, has an elderly population that constitutes a high percentage $(26 \%)$ of the total. The old Nagasaki

\footnotetext{
${ }^{4}$ These districts are the smallest unit areas of available statistical data. Other districts in the urban core of Saga have similar pyramids. The proportion of population aged 65 or over indicates $25.5 \%$ at Shirayama 2 -chome and $25.1 \%$ at Gofuku Motomachi respectively.
} 
Road runs through these inner districts, wherein the CBD of Saga is located. Outside of this core, moving outwards from two to five kilometers, the percentage of elderly in the population declines gradually all the way to the suburbs; in these areas the ratio of single to multifamily units is also high. Around the city boundary, finally, the percentage of elderly in the population begins to increase again and then remains constant where agricultural land use predominates. This profile of the distribution of the elderly in the population in Saga results primarily from the outflow of single family units from the city center.

Figure 2: Land use in Saga (2000)

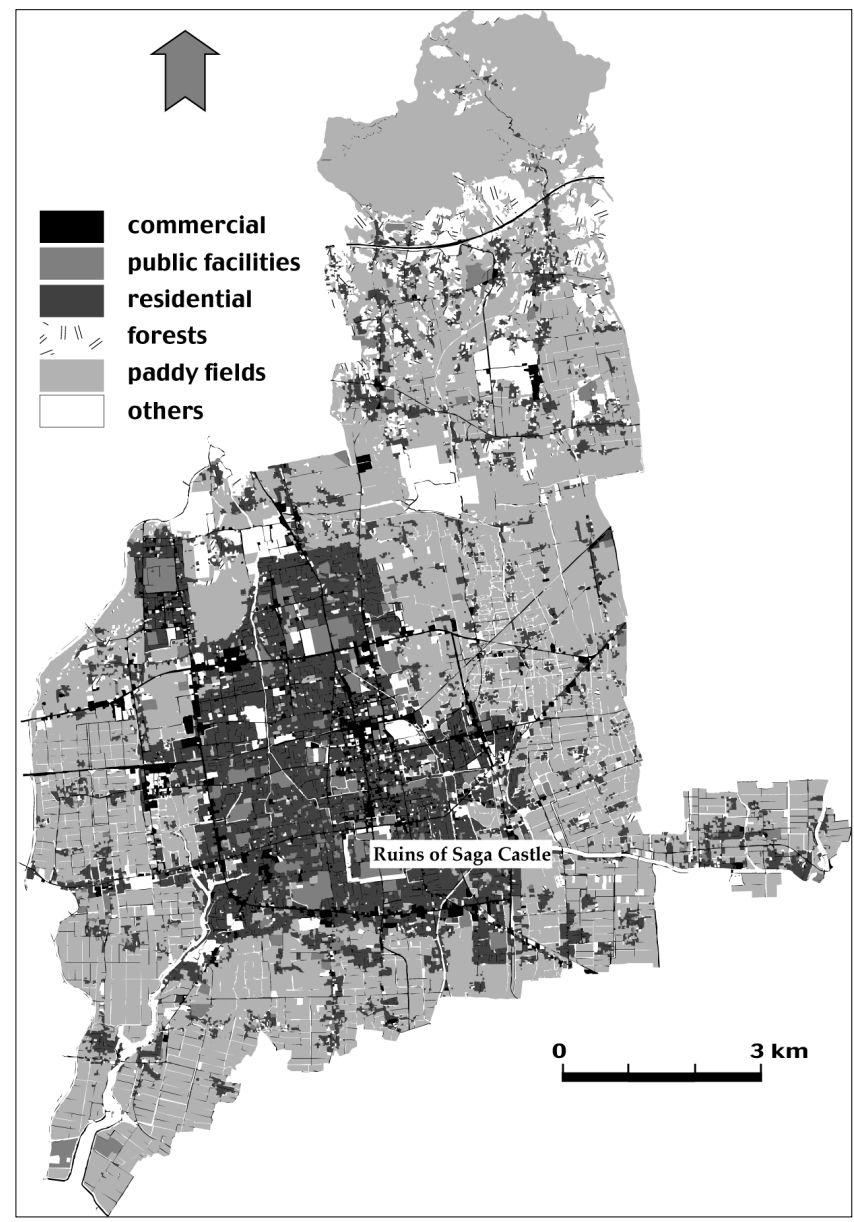

For insight into the aging of the downtown, let us consider the representative district of Chuo-honmachi, the population pyramid of which for 2000 is shown in Figure 4. Two hundred people reside in this district, including 77 males and 123 females. Of the total, 63 people, or $31.5 \%$, are aged 65 or over. People of both sexes aged 25 to 39 are scarce in the district, as is true of downtown Saga in general. 
Figure 3: Proportion of population aged 65 or over in Saga (2000)

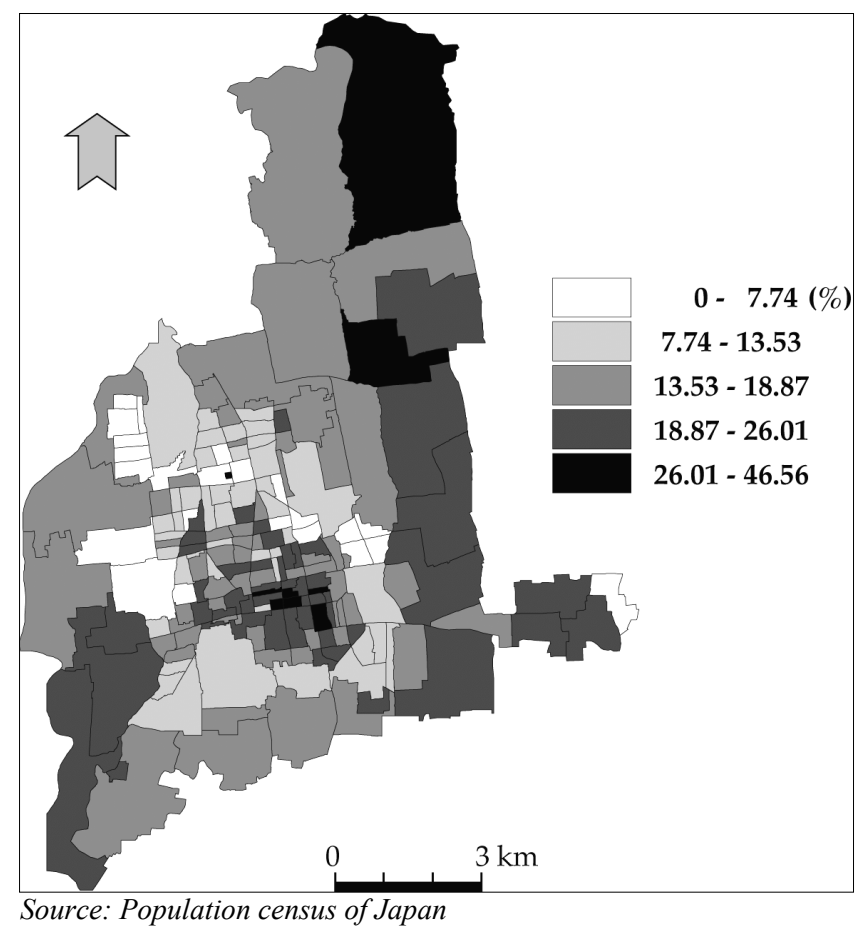

Figure 4: Population pyramid of the downtown Saga (2000)

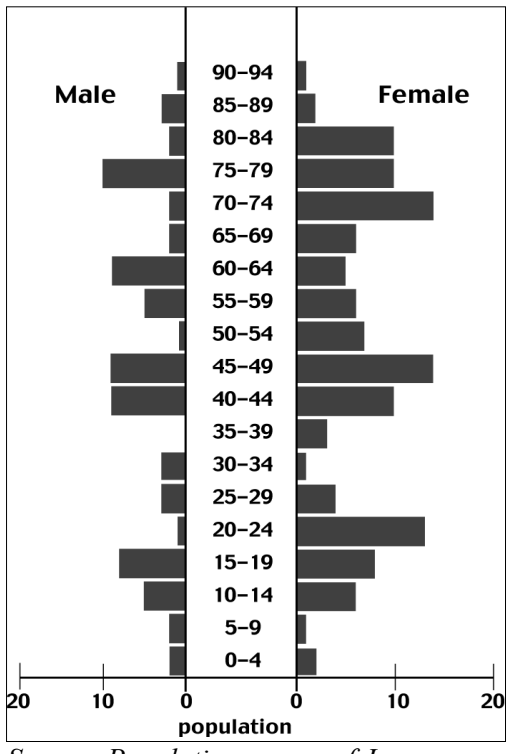

Source: Population census of Japan 
Changes in pedestrian flows ${ }^{5}$ in the Saga shopping malls since 1967 -- as represented by flows per day at three sites: (A) Plaza 656, (B) Fukuoka City Bank, and (C) Tamaya Department Store -- are shown in Figure 5. Clearly, pedestrian flows have long been on the decline at all three sites, and the decreases in pedestrian flows have been particularly pronounced in recent years. The three sites recorded a maximum flow of 36,893 in 1976, but by the latest survey the combined annual flow had shrunk to 12,567 pedestrians, a decrease of $65.9 \%$. Of the three sites, site A showed the greatest decrease in pedestrian flow with a drop 8,387 (-80.5\%). Site $C$ showed a mild decrease with a drop of $-55.9 \%(-8,259)$.

The different rates of decline at these sites have resulted from the differing circumstances of the shopping malls in which they are located. Site A's mall, for example, has been cut off from the area of the original Nagasaki Road due to the redevelopment project described below. In contrast, site C -- which has seen the smallest decrease of the three sites -- is conveniently situated on the current main avenue.

\section{Figure 5: Pedestrian flows in the Saga shopping malls}

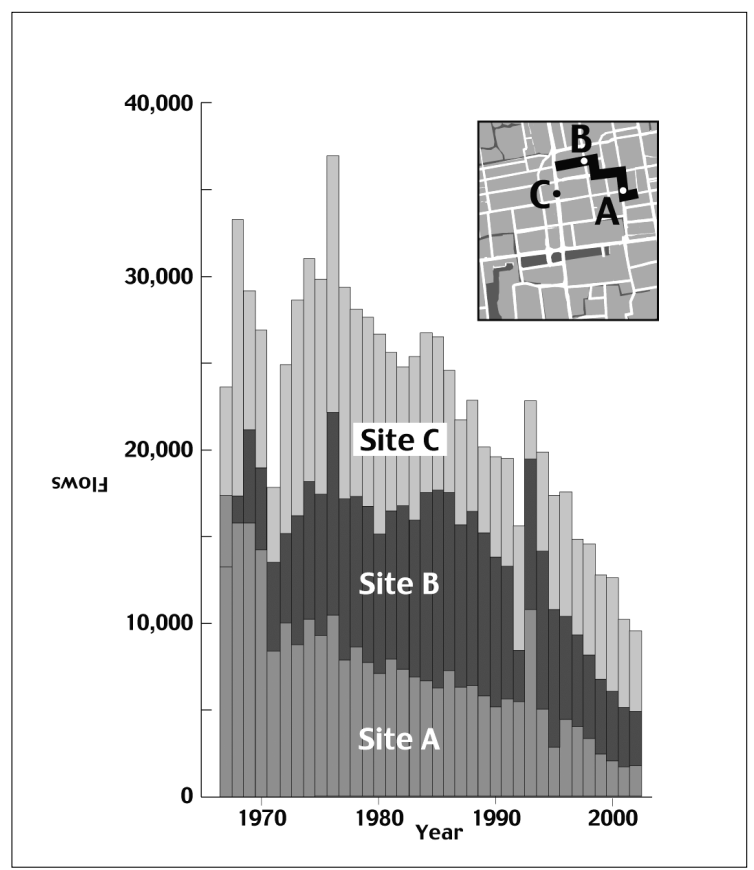

Source: Saga CCI

Site A: Plaza 656

Site B: Fukuoka City Bank

Site C: Tamaya Department Store

\footnotetext{
${ }^{5}$ Saga commercial high school students have undertaken and reported on intensive surveys of pedestrian movements throughout the downtown of Saga since 1965. Measuring pedestrian flows within the downtown and comparing them are vital tools to assess the value of downtown. The students have taken place surveys from 9 a.m. to 6 p.m. on contiguous four days including both Saturday and Sunday.
} 
Yet the significant downturn in pedestrian flows began before the suburban development projects of the latter half of the described below. Moreover, hypermarkets in the suburbs, which might seem likely causes of decreased flows, have opened only in the last year or two. Ultimately, the decline in pedestrian flows in the Saga shopping malls results from problems with the malls themselves such as lack of customer service, enfeeblement of merchant will, and small investments in their shops.

Shop vacancies are at the heart of the decline of downtown Saga. There are numerous vacant shops in the Saga shopping malls (Figure 6). The maps show the distributions of vacant shops in the malls in 1992 and 2003. In 1992, shop vacancies numbered 16. By 2003, the total had increased to 47 . The widespread vacancies that have characterized the recent economic recession have included many scales of shop -- from major large-scale retail stores in Japan such as Daiei to small stores. Most of the businesses that left shops vacant were closed due to poor sales. The southernmost mall, the Gofuku-machi shopping mall, has seen a particularly sharp increase in shop vacancies, with a 2003 vacancy rate of $28 \%$.

Figure 6: Increase of vacant stores along the Saga shopping malls

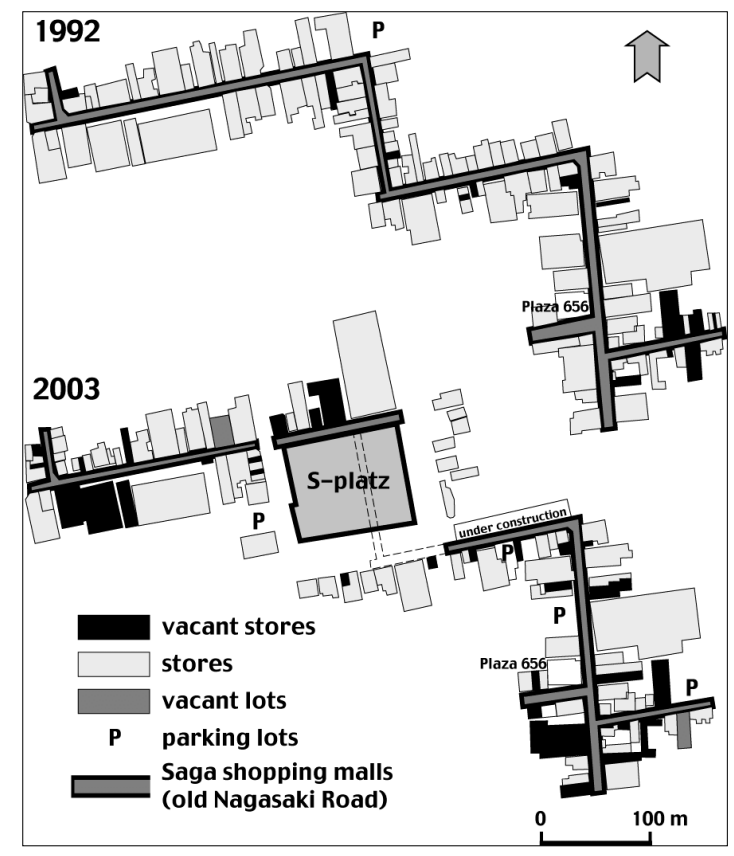

\section{Factors contributing to the decline of downtown Saga}

A number of factors have contributed to the high shop vacancy rates in downtown Saga, which fundamentally exemplify the overall decline of the downtown. First, commercial activity within Saga is so limited that the city falls within the trade area of Fukuoka, a major regional city located about 60 kilometers to the northeast. The travel time between Fukuoka and 
Saga is only about half an hour by limited express train; on holidays, many of Saga's inhabitants visit Fukuoka, where higher-order goods and services are readily accessibly.

Secondly, hyperstores and other large stores have opened in the suburbs, as mentioned above; Figure 7 shows the distribution of these stores around Saga. Two hyperstores that have opened recently; one is the AEON Shopping Town Yamato, which incorporates 40,233 square meters of floor space and 2,600 parking spaces, and the other is the Mallage SAGA, which incorporates 37,100 square meters of floor space and 3,008 parking spaces. Such commercial complexes threaten to overwhelm because the floor space of these two hyperstores is worth $45.7 \%$ of all that in the city of Saga.

Thirdly, although sufficient parking is available in the downtown area, it is neither free nor readily accessible. According to market research (Iwanaga, 2000; 2002), this poor accessibility becomes the weakest point of the downtown.

Finally, reflecting a general trend in Japan that is a serious problem for downtowns such as that of Saga, numerous shop owners are unwilling to find new tenants to occupy vacant shops. Owners of vacant shops increasingly prefer them to remain vacant rather than renting them to others; such owners have sufficient savings to support themselves and simply want to avoid the difficulties entailed in renting out their shops.

Figure 7: Distribution of large stores in Saga (2003)

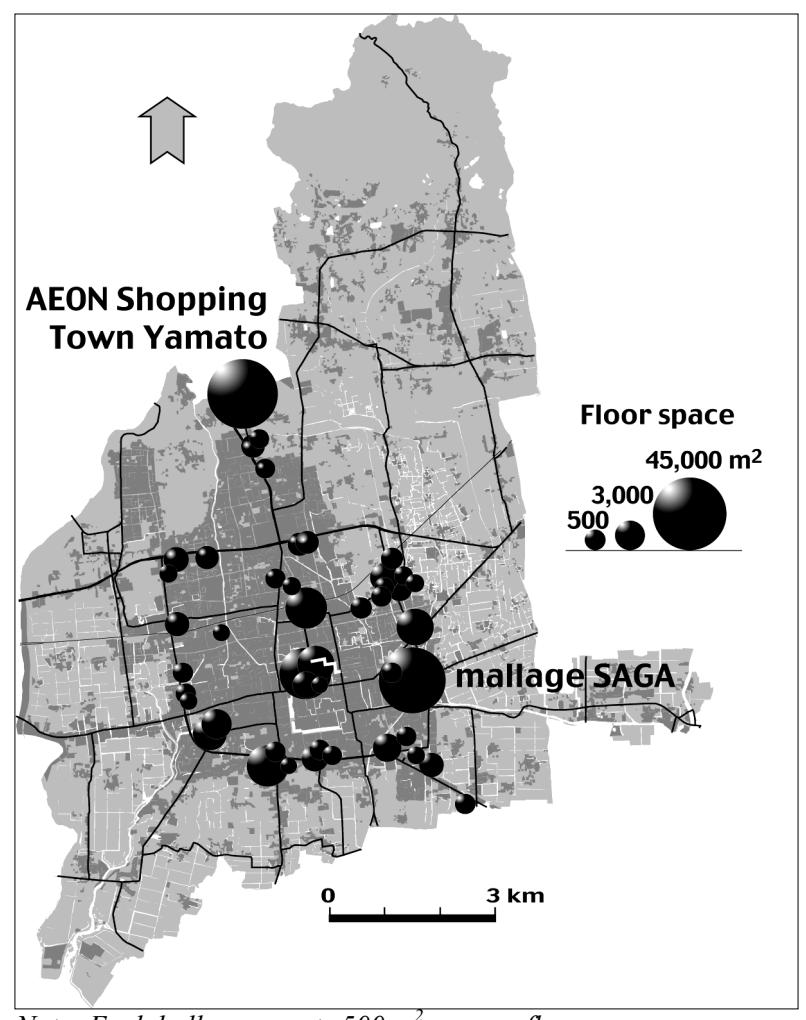

Note: Each ball represents $500 \mathrm{~m}^{2}$ or over floor space 


\section{REVITALIZATION EFFORTS IN DOWNTOWN SAGA}

While significant new commercial complexes emerged in the suburbs, as mentioned above, a major revitalization project was also undertaken in downtown Saga; this section reviews the history of this project. In 1995, the Saga Central District Redevelopment Project Association was established to make plans and inaugurate the revitalization of the downtown. The municipal government of Saga played a central role in the revitalization effort. However, in 1996 a so-called Daisan sector kaisha ${ }^{6}$, or 'third sector company' -- that is, an organization established through the cooperation of municipal and private companies -- was established to manage the centerpiece of the project, the redevelopment of a large mixeduse building to be called the "S-platz," as well as the improvement of basic services in the downtown. This public-private consortium, in which local jurisdictions are shareholders, also financed the project. The Daisan sector kaisha was authorized as a Town Management Organization (TMO), as discussed below, in October 1998, and thereafter actively managed public affairs relating in particular to the S-platz, ostensibly with public participation.

The twelve-story S-platz opened in April 1998. A supermarket moved in on the first floor, serving as an anchor tenant, a number of smaller stores were located on the second floor, a city hall sub-office was located on the third floor, and apartments occupied the fourth to twelfth floors. However, tenants had been found for only $70 \%$ of the second-floor commercial space by the opening of the building. Furthermore, overall sales for tenants subsequently fell far below predictions, and a number of tenants had to withdraw from the S-platz for this reason. In July 2001, the Daisan sector kaisha filed for corporate bankruptcy. S-platz was closed in February 2003.

The total investment by the central and municipal governments in efforts to revitalize downtown Saga exceeded ten billion yen ( $\$ 83$ million), including road maintenance and other infrastructure improvements around the downtown area. The basic premise underlying the revitalization projects attempted was that improved car access would positively influence purchasing behavior. This premise proved ill-conceived, however, because in the shopping malls along the old Nagasaki Road, purchasing behavior by pedestrians had long predominated. Revitalization efforts did little to replace the amenities for pedestrians that had largely disappeared from the downtown.

Yet other causes for the failure of revitalization efforts can also be identified: there was little public participation in decision-making processes within the revitalization project, and moreover, the Daisan sector kaisha was an irresponsible organization.

\section{REVITALIZATION AND PUBLIC PARTICIPATION}

Although public participation in efforts to revitalize downtown Saga was inadequate, municipal governments in Japan have recently begun to encourage citizens to take part in pol-

\footnotetext{
${ }^{6}$ These public-private initiatives, known as Daisan Sector Kaishas, are common throughout Japan. Daisan signifies "third," and the third sector is a uniquely Japanese concept with a significant economic impact, operating in conjunction with the first (public) and the second (private) sectors of the economy.
} 
icy formation. This trend towards aggressive town management with public participation represents a major change in Japanese public administration. This section offers a comparative discussion of existing management approaches vis-à-vis public participation in the United Kingdom, the USA, and Japan.

\section{Town Centre Management and Business Improvement Districts}

The Town Centre Management (TCM) approach was developed in the UK in the 1980s primarily for the purpose of counteracting the adverse effects of commercial growth in the suburbs by focusing on promoting downtown vitality. According to the web page of the Association of Town Centre Management, the aim of TCM is to improve the lives of real people through the enhancement of public resources, the physical and economic regeneration of urban centers, and the creation and maintenance of healthy business environments. Town Centre Management organizations are now at work across the UK in over three hundred towns and cities.

To achieve its aims, TCM encourages stakeholders and local businesses to work together in partnership. In the TCM approach, a Town Centre Manager and a few Town Centre Coordinators, acting as interfaces between the business community and local authorities, lead a Town Center Management Initiative. Initiatives encourage businesses to participate actively in the marketing, promotion, and development of their town centers.

Another successful town management approach has been developed in the USA, namely, the Business Improvement District (BID) approach. BIDs are independent, not-forprofit organizations that range in scale from major organizations such as the Times Square Business Improvement District in New York City to more limited organizations in small towns.

The Times Square BID, one of largest, exemplifies the BID approach. Established in 1992, the BID provides varied services supplementing municipal services, promotes the district, advocates for local business interests, provides information to enhance economic development, and undertakes public improvements aimed at making Times Square clean, safe, and friendly. The annual budget of the Times Square BID amounts to \$6 million; commercial property owners within the BID pay mandatory fees of approximately $0.3 \%$ of the assessed value of their property, whereas residential property owners pay $\$ 1.00$ per year.

Both TCM and BID are designed to join together businesses, property owners, cultural institutions, social service providers, government agencies, and elected officials in united efforts to revitalize their downtowns. The participation of the public as well as of business owners in decision-making processes has been vitally important to the success of both of these approaches.

\section{TMO in Japan}

Until 1998, a characteristically top-down approach has been taken in the management of municipal affairs in Japan. Under this approach, which dates from the beginning of the Meiji Period in 1868, government affairs were conducted from the government's point of view; 
however, with rapid changes in the economic and social conditions of urban areas in recent years, municipalities have had to adopt a new approach to urban planning which incorporates public participation. This approach was instituted by the Three Urban Development Laws of 1998, of which the Central Urban Area Revitalization Law is the most significant. This shift represents a dramatic change in the basic concept of urban development in Japan.

The new approach has presented extraordinary challenges both to urban planning consultants, who have only about a ten-year history of practicing independently in Japan, and local governments. First, one consequence of the old top-down urban development approach was that little expertise on urban issues was developed among such non-governmental organizations as citizens' groups and chambers of commerce. More generally, such organizations have not developed effectiveness to play significant roles in Japan.

To address these challenges, municipalities have established Town Management Organizations (TMOs), urban development organizations generally responsible for promoting urban development. A total of 268 TMOs are now operating in Japan, but among these there have been few examples of success for the reasons of insufficient expertise and time mentioned above.

Significant differences can be identified between TMOs and TCMs or BIDs. First, the Japanese and British and American approaches differ in the clarity of purpose underlying their policies. Whereas the TCM and BID approaches identify and solve issues by building consensus among interested parties, TMOs in many cases have proceeded with downtown revitalization efforts without consensus. Given the relative success of each approach, it is important that well-defined citizens' organizations need to be linked in networks with TMOs, and that information needs to be freely disseminated between them.

Secondly, TMOs, TCMs, and BIDs differ in the independence of their revenue sources. The revenue sources of TCMs and BIDs are independent and internal to the districts where the organizations operate, but TMOs continue to be funded by governments, and this fosters a dependence that entails little incentive for success.

\section{CONCLUSIONS}

In response to dynamic changes in their economic, social, and legislative circumstances, the downtowns of municipalities in Japan are forced to change their structure; downtown revitalization has become one of the most pressing concerns for municipalities in Japan. The decline of downtown Saga is typical of small cities in Japan, as are Saga's struggles with urban issues, and particularly its efforts at urban revitalization.

This study has examined the case of downtown Saga's recent decline and the failure of its attempted revitalization efforts, characterizing the complexity of the problems facing the city, and identifying factors that contributed to its lack of success in revitalizing its downtown.

One such factor was a lack of public participation. Almost no meaningful pursuit of consensus between the municipal government and citizens was conducted. The revitalization process did not reflect the ideas or views of citizens. 
Another such factor was a lack of a clear purpose in revitalization policy. The municipal government adopted its traditional top-down approach in pursuing its revitalization project to execute the budget provided by the central government.

It is vitally important that innovative ideas and proposals from citizens be incorporated in revitalization programs. Empowered to participate in urban revitalization, citizens will not only spur innovation in municipal planning and regulatory systems, but more generally will contribute to restoring the creative energy that will ensure our common future.

\section{References}

Association of Town Centre Management, URL: http://www.atcm.org City of Saga, 2001, Impact of large stores on the downtown of Saga. Saga, 217p.

Iwanaga, T., Shinohara, A., and Yamasaki, Y., 2000, Retail structure in Saga Prefecture. CRES Report Saga University, 5, 41p.

Iwanaga, T., Tsutsumida, M., and Nonaka, T., 2002, A report on Saga shopping malls. CRES Report Saga University, 9, 51p.

The BID (Times Square in NYC), URL: http://www.timessquarebid.org/the_bid/index.htm Yamashita, M., 2002. Extensive Land Use Dynamics in Tokyo. Kim, I., Nam, Y., Choi, J. eds: Diversity of Urban Development and Urban Life. Seoul, Seoul National University Press, p.197-209. 